\title{
Comparative study of linearly and circularly polarized light in polarization-sensitive optical coherence tomography
}

\author{
Wen-Chuan Kuo ${ }^{1,3}$, Chih-Ming Lai ${ }^{2}$, Chien Chou ${ }^{3,4}$, Huan-Jang Huang ${ }^{3}$, Gwo-Jen Jan ${ }^{1}$ \\ ${ }^{1}$ Department of Electrical Engineering, National Taiwan University, Taipei 106, Taiwan \\ ${ }^{2}$ Department of Electronic Engineering, Ming-Chuan University, Taoyuan 333, Taiwan \\ ${ }^{3}$ Institute of Radiological Sciences, National Yang-Ming University, Taipei 112, Taiwan \\ ${ }^{4}$ Institute of Biophotonics Engineering, National Yang-Ming University, Taipei 112, Taiwan \\ Phone: 886-2-23635251exp.305, Fax: 886-2-28815610, E-Mail: d90921018@ntu.edu.tw \\ Abstract - We report a method for extracting the birefringence properties of samples with a novel proposed
polarization-sensitive optical coherence tomography (PS-OCT) using linearly polarized incident light.
}

\section{INTRODUCTION}

Conventional PS-OCT using circularly polarized light and provides the overall phase retardation image, based on the assumption that the optical axis remains constant[1]. However, previous study has demonstrated that linearly polarized light can maintained preferentially over circularly polarized light in more dense tissues such as tendon, artery ...etc.[2]. In this study, we propose a PS-OCT setup whose incident polarization state is linearly polarized and discuss the characteristics of this new configuration.

\section{MATERIALS AND METHODS}

An $837 \mathrm{~nm}$ superluminescent diode light source, after being vertically polarized, illuminates in a Michelson interferometer where it is split by a beam splitter (BS) into a reference and a sample beams. One quarter wave plate (QWP), which oriented with its fast axis at $22.5^{\circ}$ to the horizontal axis, is inserted in reference arm of this setup. After light reflecting from the sample and recombination with the reference beam, both horizontal and vertical components are directed towards a two-channel detection unit. From AC coupling of the detector signal, the full interferometric signal is recorded. An algorithm [3] previous reported is used to determine the amplitude $\mathrm{A} i(\mathrm{z})$ and the phase $\Psi i(\mathrm{z})$ of the oscillating term of each interference signal at any depth. $i=1,2$ for the horizontal and vertical polarization state, respectively. Three parameters (reflectivity $R(z)$, phase retardation $\Phi(\mathrm{z})$, and fast axis orientation $\beta(\mathrm{z})$ of sample are calculated based on the amplitude and the phase of the interference signal with only one A-scan per measurement location as follows:

$$
\begin{gathered}
R(z) \propto A_{1}(z)^{2}+A_{2}(z)^{2} \\
\Phi(z)=\sin ^{-1}\left(\frac{\alpha^{2}+\cot ^{2}(\Delta \Psi)}{1+\cot ^{2}(\Delta \Psi)}\right)^{1 / 2} \\
\beta(z)=\frac{1}{2} \sin ^{-1}\left(\frac{1+\cot ^{2}(\Delta \Psi)}{1+\left[\frac{\cot (\Delta \Psi)}{\alpha}\right]^{2}}\right)^{1 / 2}
\end{gathered}
$$

where $\alpha=\gamma / \sqrt{\gamma^{2}+1}, \gamma=A_{1} / A_{2}$, and $\Delta \Psi=\Psi_{1}-\Psi_{2}$.

\section{RESULTS AND DISCUSSION}

Figure 1 (a) and (b) show the simulated results of measured phase retardation $\Phi_{\text {mea }}$ and fast axis angle $\beta_{\text {mea }}$ at any given value of $\Phi$ and $\beta$ of sample in this novel PS-OCT system. The dynamical range of this system is $0^{\circ}<\Phi<90^{\circ}$ and $0^{\circ}<\beta<45^{\circ}$. In order to evaluate this method, we measure a zero order QWP in front of a mirror as the linear birefringence sample. The measured results of $\Phi_{\text {mea }}$ and $\beta_{\text {mea }}$ when the azimuth angle of QWP is rotated from 0 to $180^{\circ}$ are shown in Fig. 1(c) and (d). There is a jump of $\Phi_{\text {mea }}$ observed in Fig. 1 (c) occurred at $\beta$ rotated near $90^{\circ}$ that is agree with the simulated results in Fig. 1(a). Besides, $\frac{\partial \Phi}{\partial \gamma}$ and $\frac{\partial \Phi}{\partial(\Delta \Psi)}$ at any given value of $\Phi$ and $\beta$ are calculated in Fig. 2 (a) and (b) separately. In conventional PS-OCT system, the measurement sensitivity is a constant at any $\Phi$ and $\beta$ of tested sample. While in Fig. 2, it represents the measurement sensitivity of this novel PS-OCT system which is dependent on the $\Phi$ and $\beta$ of tested sample. Therefore, the measurement sensitivity can be optimized by orienting one major axis of the tissue proper angle relative to the incident linear polarization axis. 


\section{THP-35(T7)}

\section{CONCLUSION}

In conclusion, a PS-OCT system which use linearly polarized incident light is setup and verified successfully. This novel proposed method can provides backscattered intensity, birefringence, and fast axis orientation simultaneously. Besides, the fast axis orientation can be determined more reliably from using the amplitude and phase difference at the same time. Moreover, unlike conventional PS-OCT which uses circularly polarized light, the measurement sensitivity of this novel configuration can be optimized by orienting one major axis of the tissue proper angle relative to the incident linear polarization axis. Therefore, this novel method may be a potentially tool for imaging more dense scattering tissues.

\section{REFERENCES}

[1] S. Guo, J. Zhang, L. Wang, J. S. Nelson, and Z. Chen, "Depth-resolved birefringence and differential optical axis orientation measurements with fiber-based polarization-sensitive optical coherence tomography, "Opt. lett., 29(17), pp. 2025-2027 (2004).

[2] V. Sankaran, J. T. Walsh, and D. J. Maitland, "Comparative study of polarized light propagation in biologic tissues, " J. Biomed. Opt., 7(3), pp. 300-306 (2002).

[3] C. K. Hitzenberger, E. Gotzinger, M. Sticker, M. Pircher and A. F. Fercher, "Measurement and imaging of birefringence and optic axis orientation by phase resolved polarization sensitive optical coherence tomography, "Opt. Exp., 13, pp. 780-790 (2001).

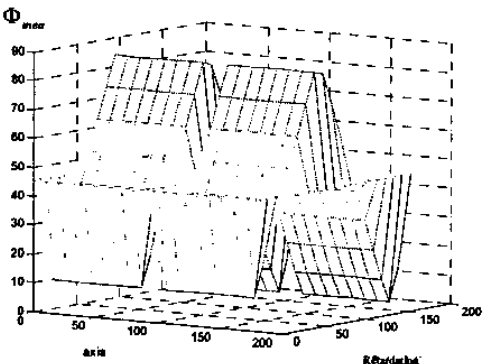

(a)

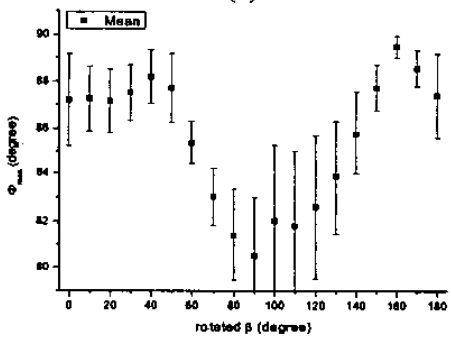

(c)

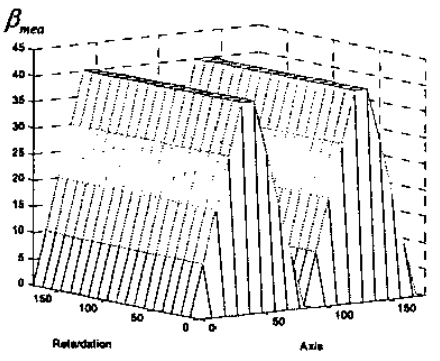

(b)

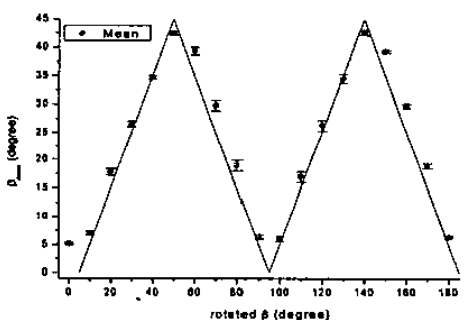

(d)

Fig. 1 The simulated and experimental results of $\Phi$ and $\beta$ in a tested sample.

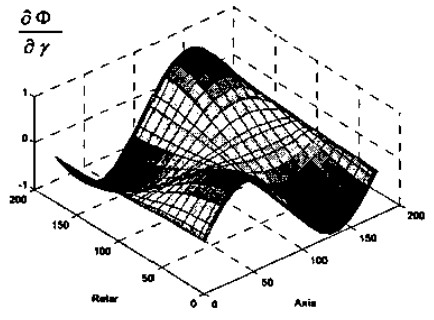

(a)

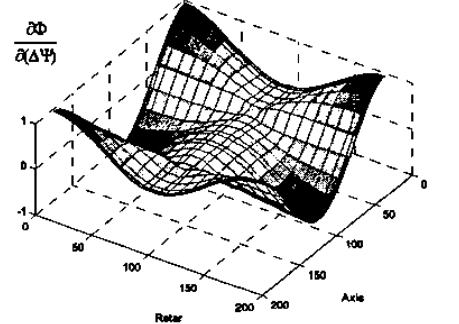

(b)

Fig. 2 The calculated $\frac{\partial \Phi}{\partial \gamma}$ and $\frac{\partial \Phi}{\partial(\Delta \Psi)}$ distribution at any given $\Phi$ and $\beta$ in a tested sample. 